\title{
A highly accurate corrected scheme in solving the Laplace's equation on a rectangle
}

Adigüzel A. Dosiyev ${ }^{1, *}$ and Hediye Sarıkaya ${ }^{1}$

${ }^{1}$ Department of Mathematics, Near East University,Nicosia, TRNC, Mersin 10, Turkey

\begin{abstract}
A pointwise error estimation of the form $O\left(\rho h^{8}\right), h$ is the mesh size, for the approximate solution of the Dirichlet problem for Laplace's equation on a rectangular domain is obtained as a result of three stage (9-point, 5-point and 5-point) finite difference method; here $\rho=\rho(x, y)$ is the distance from the current grid point $(x, y) \in \Pi^{h}$ to the boundary of the rectangle $\Pi$.
\end{abstract}

\section{Introduction}

A highly accurate corrected scheme is one of the compact approximations for decreasing the number of unknowns, that gives the effective numerical solution of differential equations.

In [1] a two-stage difference method was given on the way of solving the Dirichlet problem for Laplace's equation on a rectangular parallelepiped. It was assumed that the sixth order derivative of the boundary functions which are given on the faces of a parallelepiped satisfy the Hölder condition, and on the edges, besides the continuity they satisfy the compatibility condition for second derivatives, which results from the Laplace equation. It was proved that by using a simple 7-point scheme in two stages the order of uniform error can be improved up to $O\left(h^{4} \ln h^{-1}\right)$.

In this paper, a new three-stage difference method to solve the Dirichlet problem for Laplace's equation on the rectangular domain is given. By using at the first stage the 9-point, and at the second and the third stages the 5point schemes for error of the approximate solution a pointwise estimation of order $O\left(\rho h^{8}\right)$ is obtained, where $\rho=\rho(x, y)$ is the distance from the current grid point $(x, y) \in \Pi^{h}$ to the boundary of the rectangle $\Pi$. Numerical experiment is given to support the theoretical analysis made.

The weighted estimates for the approximate solutions by the finite difference method of the Dirichlet problem for the Laplace and Poisson equations, at the rate as $(\rho+h)^{\sigma} h^{2}, 0<\sigma \leq 1$, were obtained in [2]. A highly accurate finite difference approximations by correcting the right hand term using the high order differences of the numerical solution of differential equation without the weighted estimates was constructed in [3], [4].

\section{Finite Difference Problem}

Let $\Pi=\{(x, y): 0<x<a, 0<y<b\}$ be rectangle, $a / b$ be rational, $\gamma_{j}\left(\gamma_{j}^{\prime}\right), j=1,2,3,4$, be the sides, including (excluding) the ends, numbered counterclockwise begining by the left side $\left(\gamma_{0} \equiv \gamma_{4}, \gamma_{1} \equiv \gamma_{5}\right)$ and let $\gamma=\cup_{j=1}^{4} \gamma_{j}$ be the boundary of $\Pi$. Denote by $s$ the arclength, measured along $\gamma$, and by $s_{j}$ the value of $\mathrm{s}$ at the beginning of $\gamma_{j}$. We say that $f \in C^{k, \lambda}(D)$, if $f$ has $k$ th derivatives on $D$ satisfying a Hölder condition where $0<$ $\lambda<1$

We consider the following boundary value problem

$$
\Delta u=0 \text { on } \Pi, u=\phi_{j}(s) \text { on } \gamma_{j}, j=1,2,3,4,
$$

where $\Delta \equiv \partial^{2} / \partial x^{2}+\partial^{2} / \partial y^{2}, \phi_{j}$ are given functions of $s$. Assume that

*Corresponding author: adiguzel.dosiyev@emu.edu.tr 


$$
\begin{aligned}
& \phi_{j} \in C^{12, \lambda}\left(\gamma_{j}\right), 0<\lambda<1, \quad j=1,2,3,4, \\
& \phi_{j}^{(2 q)}\left(s_{j}\right)=(-1)^{q} \phi_{j-1}^{(2 q)}\left(s_{j}\right), q=0, \ldots, 5 .
\end{aligned}
$$

On $\Pi$, we introduce the functions $u^{m}, m=4,8$ defined by

$$
u^{m}=u^{m}(x, y)=\left(\frac{1}{2}\right)\left(\left(\frac{\partial^{m} u(x, y)}{\partial x^{m}}\right)+\left(\frac{\partial^{m} u(x, y)}{\partial y^{m}}\right)\right),
$$

where $u$ is the solution of Dirichlet problem defined by (1).

It is easy to check that for each of $m=4,8$ the function (4) is the solution of following boundary value problem

$$
\begin{aligned}
& \Delta u^{m}=0 \text { on } \Pi, \\
& u^{m}=\Psi_{k}^{m}=\Psi_{k}^{m}(y) \text { on } \gamma_{k}, k=1,3, \\
& u^{m}=\Psi_{l}^{m}=\Psi_{l}^{m}(x) \text { on } \gamma_{l}, l=2,4,
\end{aligned}
$$

where

$$
\Psi_{k}^{m}(y)=\left(\frac{d^{m} \phi_{k}}{d y^{m}}\right), k=1,3, \Psi_{l}^{m}(x)=\left(\frac{d^{m} \phi_{l}}{d x^{m}}\right), l=2,4 .
$$

From (2), (3) and (8) it follows that the following $(12-m)-t h$ order derivatives are bounded on $\Pi$ :

$$
\max _{0 \leq p \leq \frac{12-m}{2}} \sup _{(x, y) \in \Pi}\left|\frac{\partial^{12-m} u^{m}}{\partial x^{2 p} \partial y^{12-m-2 p}}\right|<\infty, m=4,8 .
$$

Let $h>0, b / h \geq 8$ and $a / h \geq 8$ where $a / h$ and $b / h$ are integers. We define $\Pi^{h}$ a square grid net on $\Pi$ with step size $h$, obtained by the lines $x, y=0, h, 2 h, \ldots$. Let $\gamma_{j}^{h}$ be a set of nodes on the interior of $\gamma_{j}$, and let

$$
\gamma^{h}=\bigcup_{j=1}^{4} \gamma_{j}^{h}, \dot{\gamma}_{j}=\gamma_{j-1} \cap \gamma_{j}, \quad \bar{\gamma}^{h}=\bigcup_{j=1}^{4}\left(\gamma_{j}^{h} \cup \dot{\gamma}_{j}\right), \quad \bar{\Pi}^{h}=\Pi^{h} \cup \bar{\gamma}^{h} .
$$

Let the operator $B^{p}, p \in\{0,1\}$ be defined as follows:

$$
\begin{aligned}
B^{p} u(x, y)= & \left(\frac{1}{5-p}\right)\{u(x+h, y)+u(x-h, y)+u(x, y+h)+u(x, y-h)\} \\
& +\left(\frac{1-p}{20}\right)\{u(x+h, y+h)+u(x-h, y+h)+u(x+h, y-h) \\
& +u(x-h, y-h)\} .
\end{aligned}
$$

We consider for the approximation of problems (5)-(7) by the following system of difference equations:

$$
\begin{aligned}
& u_{h}^{4}=B^{0} u_{h}^{4} \text { on } \Pi^{h}, u_{h}^{4}=\Psi_{j}^{4} \text { on } \gamma_{j}^{h} \cup \gamma_{j}, j=1,2,3,4 ; \\
& u_{h}^{8}=B^{1} u_{h}^{8} \text { on } \Pi^{h}, u_{h}^{8}=\Psi_{j}^{8} \text { on } \gamma_{j}^{h}, j=1,2,3,4 .
\end{aligned}
$$

By the maximum principle (see[5], Chap.4), problems (11) and (12) have the unique solution.

Consider the following systems:

$$
\begin{aligned}
& q_{h}=B^{p} q_{h}+g_{h}, \text { on } \Pi^{h}, q_{h}=0 \text { on } \gamma_{h}, \\
& \bar{q}_{h}=B^{p} \bar{q}_{h}+\bar{g}_{h}, \text { on } \Pi^{h}, \bar{q}_{h} \geq 0 \text { on } \gamma_{h},
\end{aligned}
$$

where $B^{p}, p \in\{0,1\}$ is the operator defined by (10), $g_{h}$ and $\bar{g}_{h}$ are given functions, and $\left|g_{h}\right| \leq \bar{g}_{h}$ on $\Pi^{h}$. 
Lemma 1. The solutions $q_{h}$ and $\bar{q}_{h}$ of systems (13) and (14) hold the inequality

$$
\left|q_{h}\right| \leq \bar{q}_{h} \text { on } \bar{\Pi}^{h} \text {. }
$$

Proof.Lemma 1 follows by the Comparison theorem (see Chapter 4 in [5]).

In what follows and for simplicity $c, c_{0}, c_{1}, \ldots$ defined the positive constants which are independent of $h$ and the nearest factor, the similar notation is eonvenient for varied constants.

Let $\varpi$ be a solution of the problem

$$
\Delta \varpi=0 \text { on } \Pi, \varpi=\theta_{j}(s) \text { on } \gamma_{j}, j=1,2,3,4,
$$

where $\theta_{j}, j=1,2,3,4$ are given functions and

$$
\begin{aligned}
& \theta_{j} \in C^{2^{3-p}, \lambda}\left(\gamma_{j}\right), \quad 0<\lambda<1, \quad j=1,2,3,4, \quad p \in\{0,1\} \\
& \theta_{j}^{(2 q)}\left(s_{j}\right)=(-1)^{q} \theta_{j-1}^{(2 q)}\left(s_{j}\right), \quad q=0, \ldots, 3-2 p .
\end{aligned}
$$

According to Taylor formula, we have

$$
\max _{(x, y) \in \bar{\pi}}\left|B^{p} \varpi-\varpi\right| \leq c h^{2^{3-p}}, \text { where } p \in\{0,1\}
$$

where $\varpi$ is the solution of problem (15)-(17) on $\Pi^{h}$.

Lemma 2. For the solution of problem

$$
q_{h}=B^{p} q_{h}+h^{\ell} \text { on } \Pi^{h}, q_{h}=0 \text { on } \gamma_{h} \text {, where } p \in\{0,1\}
$$

the following inequality holds:

$$
q_{h} \leq \mathbb{k} \rho d h^{\ell-2} \text { on } \Pi^{h},
$$

where $\mathbb{k}$ is a positive constant defined by follows,

$$
\mathbb{k}= \begin{cases}\frac{5}{3}, & \text { if } p=0, \\ 2, & \text { if } p=1,\end{cases}
$$

$\ell>2$ is an integer, $d=\max \{a, b\}$, and $\rho=\rho(x, y)$ is the distance between the boundary of the rectangle $\Pi$ and the current point $(x, y) \in \bar{\Pi}^{h}$.

\section{Approximation of the solution using three stage method}

Lemma 3. The estimation holds

$$
\left|u_{h}^{m}-u^{m}\right| \leq c \rho h^{10-m}, \text { where } m \in\{4,8\}
$$

where $u_{h}^{m}$ is the solution of system (11) when $m=4$, and of system (12) when $m=8, u^{m}$ is the trace of exact solution of problem (5)-(7) on $\bar{\Pi}^{h}$.

\section{Proof. Let}

$$
\varepsilon_{h}=u_{h}^{m}-u^{m} \text { on } \bar{\Pi}^{h} \text {. }
$$

It is obvious that

$\varepsilon_{h}=B^{p} \varepsilon_{h}+\left(B^{p} u^{m}-u^{m}\right)$ on $\Pi^{h}, \varepsilon_{h}=0$ on $\gamma^{h}$. 
By virtue of Lemma 2, for the solution of problem (22), we obtain

$$
\left|\varepsilon_{h}\right| \leq c \rho h^{10-p} .
$$

From equations (21) and (23) follows the proof of Lemma 3.

Let $u_{h}^{4}$ and $u_{h}^{8}$ be the solutions of difference problem (11) and (12), respectively. The solution of the problem (1) on the grid $\bar{\Pi}^{h}$ is approximated by a solution $u_{h}$ of the following difference problem

$$
u_{h}=B^{1} u_{h}-\left(\frac{h^{4}}{4 !}\right) u_{h}^{4}-\left(\frac{h^{8}}{8 !}\right) u_{h}^{8} \text { on } \Pi^{h}, u_{h}=\phi_{j} \text { on } \gamma_{j}^{h}, j=1,2,3,4 \text {. }
$$

Theorem 1. On $\Pi^{h}$, the pointwise estimation is true

$$
\left|u_{h}-u\right| \leq c \rho h^{8},
$$

where $u$ is the trace of the problem defined by (1) on $\Pi^{h}, u_{h}$ is a solution of system (24).

Proof. By Taylor's formula, we have

$$
u(x, y)=B^{1} u(x, y)-\left(\frac{h^{4}}{4 !}\right) u^{4}(x, y)-\left(\frac{h^{8}}{8 !}\right) u^{8}(x, y)-r(x, y)
$$

where $(x, y) \in \bar{\Pi}^{h}, u^{4}$ and $u^{8}$ are the functions defined by (4),

$$
r(x, y)=\left(\frac{h^{12}}{2 \times 12 !}\right)\left(\left(\frac{\partial^{12} u\left(x+\theta_{1}, y\right)}{\partial x^{12}}\right)+\left(\frac{\partial^{12} u\left(x, y+\theta_{2}\right)}{\partial y^{12}}\right)\right),\left|\theta_{i}\right|<1, i=1,2 .
$$

By (9), we have

$$
|r(x, y)| \leq c h^{12} .
$$

We put

$$
\varepsilon_{h}=u_{h}-u \text { on } \bar{\Pi}^{h}
$$

where $u$ is the solution of Dirichlet problem (1) and $u_{h}$ is the solution to the system (24).

In the view of (24), (26) and (29), we get

$$
\varepsilon_{h}=B^{1} \varepsilon_{h}+\left(\frac{h^{4}}{4 !}\right)\left(u_{h}^{4}(x, y)-u^{4}(x, y)\right)+\left(\frac{h^{8}}{8 !}\right)\left(u_{h}^{8}(x, y)-u^{8}(x, y)\right)+r \text { on } \Pi^{h},
$$

$\varepsilon_{h}=0$ on $\gamma_{j}^{h}$.

By virtue of Lemma 3 and (28) it follows that

$$
\left(\frac{h^{4}}{4 !}\right)\left|u^{4}-u_{h}^{4}\right|+\left(\frac{h^{8}}{8 !}\right)\left|u^{8}-u_{h}^{8}\right|+|r| \leq c_{1} \rho h^{10}+c_{2} h^{12} \leq c_{3} h^{10} \text { on } \Pi^{h} .
$$

Now, from (30)-(32) we have

$$
\begin{aligned}
& \varepsilon_{h}=B^{1} \varepsilon_{h}+g_{h} \text { on } \Pi^{h}, \\
& \varepsilon_{h}=0 \text { on } \gamma^{h},
\end{aligned}
$$

where

$$
\left|g_{h}\right| \leq c_{3} h^{10} .
$$

Let $\varepsilon_{h}$ be a solution of the problem

$$
\varepsilon_{h}=B^{1} \varepsilon_{h}+c_{3} h^{10} \text { on } \Pi^{h},
$$




$$
\varepsilon_{h}=0 \text { on } \gamma^{h} \text {. }
$$

Then, by Lemma 1 and Lemma 3 , we obtain

$$
\left|\varepsilon_{h}\right| \leq\left|\varepsilon_{h}\right| \leq 2 c_{3} d h^{8} \rho
$$

or

$$
\left|\varepsilon_{h}\right| \leq \operatorname{ch}^{8} \rho,
$$

where $c=2 c_{3} d, d=\max \{a, b\}$, and $\rho=\rho(x, y)$ is the distance between the boundary of the rectangle $\Pi$ and the current point $(x, y) \in \bar{\Pi}^{h}$.

Remark 1. The proposed method can be generalised for the mixed boundary value problem for Laplace's equation.Furthermore, the idea of the proposed method can be applied to increase an accuracy of the finite difference approximation of $\mathrm{KdV}$ equation used in [6].

\section{References}

1. E.A. Volkov, Comp. Maths Math. Phys.49(3), 496-501(2009)

2. E.A. Volkov, Proc. Steklov Inst. Math., 117, 119-134, (1972)

3. E.A. Volkov, Differ. Equations, 1(7), 946-960, (1965)

4. G. K. Berikelashvili and B. G. Midodashvili, Differential Equations, 51(1),107-115, (2015)

5. A.A. Samarskii,The theory of difference schemes, Marcel Dekker, Inc.(2001)

6. A. Yokus and H. Bulut, Indian Journal of Physics, (5), 1-5, (2018) 